\title{
¿Entras a la cueva? Una experiencia multisensorial para trabajar las Ciencias en la etapa de Educación Infantil
}

\author{
Ester Mateo González, Luis Miguel Ferrer Bueno, Beatriz Mazas Gil \\ y Esther Cascarosa Salillas \\ Departamento de Didácticas Específicas. Área de Didáctica de las Ciencias \\ Experimentales. Universidad de Zaragoza. Zaragoza (España)
}

[Recibido el 9 de octubre de 2019, aceptado el 8 de mayo de 2020]

Este trabajo muestra el diseño, planificación, implementación en el aula y análisis de una experiencia multisensorial dirigida a niños de Infantil en la que se trabajan contenidos científicos. El primer objetivo consiste en educar sensorialmente al alumnado mediante una exploración en la que utilizan los cinco sentidos de manera simultánea a la vez que disfrutan de las sensaciones que ofrece la manipulación de materiales naturales. El segundo objetivo es que los maestros valoren positivamente la educación sensorial en ciencias y reconozcan su insustituible valor didáctico en el proceso de enseñanza-aprendizaje. Se analiza cómo la actividad permite al alumnado experimentar, sentir, pensar y describir las propiedades de los materiales de su entorno inmediato a través de los cinco sentidos paralelamente. Finalmente, se aporta vocabulario científico para trabajar la educación sensorial con el objetivo de que los niños sean capaces de describir objetos o sensaciones en otros contextos.

Palabrasclave: Educación Infantil; enseñanza de las Ciencias; educación sensorial; Universidad-escuela.

\section{Would you enter the cave? A multisensory experience in early childhood education to work Sciences}

This work shows the design, planning, classroom implementation and analysis of a multisensory experience where scientific contents are worked with children of Early Childhood Education. The first objective is to educate students by means of an exploration experience in which they use the five senses in a simultaneous way and they enjoy with the sensations offered through the manipulation of natural materials. The second aim is to achieve that teachers value the sensory education in science in a positively way and they recognize its irreplaceable didactic value in the teaching-learning process. This work is analysed studying how the activity allows students to experiment, to feel, to think and to describe the properties of the materials of their immediate environment through the five senses. Finally, a proposal of scientific content is provided to work sensory education, trying to increase the possibilities of exploration and the sensory vocabulary of both, students and teachers, for describing objects and sensations in other contexts.

Keywords: Early childhood education; Science teaching; sensory education, University-school initiative.

Para citar el artículo. Mateo González, E., Ferrer Bueno, L.M., Mazas Gil, B. y Cascarosa Salillas, E.(2020). ¿Entras a la cueva? Una experiencia multisensorial para trabajar las Ciencias en la etapa de Educación Infantil. Ápice. Revista de Educación Científica, 4 (2), 51-62. DOI: https://doi. org/10.17979/arec.2020.4.2.5755

Contacto.emateog@unizar.es 


\section{Introducción y marco teórico}

La etapa de Educación Infantil (EI) es el periodo en el que comienza el desarrollo personal, cognitivo, físico, lingüístico, afectivo y social del ser humano. Este desarrollo es un proceso largo que abarca toda una vida pero los primeros años son decisivos en la formación de las personas, ya que en ellos se asientan los rasgos de personalidad y se adquieren destrezas y capacidades en las que se apoyan aprendizajes posteriores (Diez Navarro, 2013).

En esta etapa, los niños/as establecen su primer contacto con los fenómenos naturales. Infinidad de vivencias les rodean, como un rayo de sol que entra por la ventana, una brisa de aire que refresca en el parque, un olor delicioso al entrar en la cocina, el tacto suave de un peluche, etc. Los niños/as presentan un interés natural por conocer estos sucesos, relacionarse con ellos y poner en juego sus propias capacidades para interactuar con estos elementos y dar explicaciones. Son el colectivo más receptivo a aprender cosas nuevas que existe (Vega, 2012). Desde esta perspectiva, es necesario considerar El como una etapa donde es necesario favorecer y reforzar el potencial y desarrollo pleno de los niños/ as, así como su manera de descubrir y relacionarse con el mundo.

La base del aprendizaje en los primeros años de los niños/as es en gran medida perceptual: necesitan llenarse de sensaciones y conocer el mundo que les rodea a través de los sentidos: tocar, lamer, escuchar, observar y olfatear (Vila y Cardo, 2012). Estas acciones son el primer paso para comprender lo que ocurre a su alrededor. Además, la percepción a través de los sentidos siempre va unida a sensaciones y sentimientos: de esta manera, no captamos el mundo como es, sino como lo interpretamos: con placer, con dolor, con alegría, con rabia, con repugnancia, etc. Por ello, es necesario trabajar las emociones unidas a las percepciones compartiendo e interiorizando dichos sentimientos (De Puig, 2004).

Las emociones tanto en el ámbito educativo en general (Gardner, 2005) como en el del aprendizaje y la enseñanza de las ciencias en particular, adquieren un papel cada vez más estudiado y destacado. Es necesario incluir las emociones en el propio aprendizaje de los pequeños para que sean capaces de reconocerlas, controlarlas y autorregularlas (Mellado, Borrachero, Brígido y Melo, 2014).

Posteriormente, el pensamiento se puede construir gracias a la percepción del mundo exterior y gracias al sentimiento que provoca esa percepción. Sentidos y pensamiento interactúan: las percepciones actúan de material en bruto que ha de ser examinado, almacenado y organizado por el intelecto y a la vez los pensamientos influyen en lo que vemos. Finalmente, el lenguaje permite analizar lo que los sentidos nos presentan para darle sentido y significado (De Puig, 2004).

Aunque el punto de partida para promover la construcción científica nace de la curiosidad que todos tenemos por comprender los fenómenos que nos rodean, esta predisposición no adquiere su efecto por sí sola (Mazas, Gil-Quílez, Martínez-Peña, Hervás y Muñoz, 2018). Si enseñamos a los niños/as a ver el mundo a través de la Ciencia desde los primeros años de su formación escolar, estaremos ayudándoles a construir explicaciones del mundo basadas en criterios científicos (Driver, Guesne y Tiberguien, 1989; GarcíaCarmona, Criado y Cañal, 2004). El desarrollo de este tipo de pensamiento requiere la adquisición de procedimientos y actitudes concretas, como la observación, la comparación, la clasificación, la experimentación, el razonamiento o la curiosidad las cuales enriquecen la capacidad del niño/a para relacionarse y ordenar su realidad más cercana.

En el currículo oficial de El (Real Decreto 1085/2008), la educación sensorial se trabaja específicamente desde el área del conocimiento de sí mismo y autonomía personal (en el bloque "El cuerpo y la propia imagen") en el 10 y en el 2 을 ciclo y desde el área de conocimiento del entorno (en el bloque "Medio físico: elementos, objetos y materiales") en el 1 o 
ciclo. El alumnado tiene que identificar y utilizar los sentidos y expresar verbalmente las sensaciones y percepciones adquiridas. En el currículo se propone trabajar a través de los sentidos en la exploración y manipulación de los objetos y materiales de su entorno inmediato y comparar objetos en función de alguna de sus propiedades fomentando el interés por la exploración. Aunque debido a que uno de los principios que se marcan en el currículo es la globalización, será un contenido que se trabajará de manera continua ya que los niños/as aprenden estableciendo relaciones entre conceptos, percibiendo las cosas como totalidades o globalidades que, si se descomponen, pierden su sentido (Fernández y Bravo, 2015). Desde esta perspectiva, la enseñanza en Ciencias tiene un enfoque globalizador donde los alumnos aprenden desde una perspectiva integradora y diversa.

Dada la importancia que tiene utilizar los sentidos durante los primeros años de escolarización, es vital por parte de los maestros/as plantear experiencias donde se aproveche la necesidad que tiene el alumnado de explorar el mundo que les rodea de forma sensorial. Los sentidos son estimulados y provocados espontáneamente, pero también es necesario proponer actividades previamente planificadas para que sean realmente provechosas y útiles para su aprendizaje. Además, es importante crear ambientes ricos en estímulos sensoriales ya que los niños/as pueden comprender mejor las informaciones que les llegan por varios canales sensoriales (Westerhoff, 2010). De esta manera, entendemos que en una experiencia multisensorial los alumnos trabajan con los cinco sentidos simultáneamente con el objetivo principal de percibir y sentir el mundo que nos rodea dándole sentido y significado en un contexto concreto.

A pesar de reconocer la necesidad de promover la alfabetización científica desde El, existe un déficit de trabajos de investigación en Didáctica de las Ciencias en esta etapa debido, entre otros factores, a la dificultad que supone investigar con niños/as entre 0 y 6 años por sus limitaciones lingüísticas y a la escasa importancia social y académica con la que se proyecta esta etapa en la sociedad (Cantó, de Pro y Solbes, 2016; Borgerding y Raven, 2016; Montiera y Jiménez-Aleixandre, 2016).

\section{Objetivos}

Los principales objetivos de este trabajo son:

- Diseñar e implementar una actividad multisensorial donde se trabajan contenidos científicos. De esta manera, se pretende contribuir al aprendizaje científico de los niños/as de El trabajando aspectos relacionados con el medio natural a través de los sentidos para comprender el entorno que nos rodea: reconociendo y compartiendo sensaciones y emociones, y dándole sentido y significado.

- Analizar las transcripciones de las conversaciones de los niños/as para detectar si reconocen los cinco sentidos como herramientas fundamentales para explorar el entorno que nos rodea.

- Reflexionar con las maestras de El implicadas sobre la educación sensorial en Ciencias y su valor didáctico en el proceso de enseñanza y aprendizaje.

\section{Contexto y metodología}

Esta actividad se lleva a cabo gracias a la colaboración entre los profesores del área de Didáctica de las Ciencias Experimentales de la Universidad de Zaragoza con las maestras del colegio público CEIP Fernández Vizarra (Monzalbarba, Zaragoza) desde hace varios años. Al empezar el curso, se estableció una secuencia de actividades para trabajar el tema de la luz a lo largo de todo el curso. 
Tras los primeros días de proyecto y en base a la pregunta de un alumno "¿Qué pasaría si no hubiera luz?", se diseñó una actividad multisensorial en la que los niños/as entrasen a oscuras a una clase que se había decorado como si fuera el interior de una cueva. En ella se habían preparado diferentes espacios donde, libremente, podrían manipular elementos de la Naturaleza que se pueden encontrar en una cueva. Esta actividad sirve a las maestras como punto de partida para posteriormente trabajar cómo influye la luz en cada uno de los elementos que se introdujeron en la experiencia: la luz en las plantas, la luz que produce el fuego, la luz en nuestro organismo, el color, brillo y diafanidad de los minerales, etc.

La actividad se realizó con 12 alumnos/as de 2으 de El y 21 alumnos/as de 3 de El (4-6 años). Se dividieron en 3 grupos internivelares. Cada grupo realizó la experiencia multisensorial durante 20 minutos. Además, se realizó una sesión de 1 hora de reflexión del grupo completo con las dos maestras. En esta sesión se preguntó oralmente a todos los alumnos: ¿Cómo te has sentido? y ¿qué es lo que más te ha gustado? Posteriormente, se pidió a las maestras una valoración escrita de la actividad.

La metodología utilizada en este trabajo tiene un corte cualitativo (Hernández-Sampieri, Fernández y Baptista, 2006), donde los datos son perspectivas y puntos de vista de las personas que participan, recogidos mediante observación, registro fotográfico y grabaciones de vídeo y audio y entrevistas al grupo y a las maestras. A partir de las grabaciones de vídeo y audio se han realizado transcripciones de la actividad multisensorial y de las reflexiones posteriores, de las cuales se han extraído los intereses, emociones y contenidos científicos que el alumnado expresaba en sus verbalizaciones y el análisis de las dos maestras de El implicadas en el proyecto.

\section{Resultados}

\section{Desarrollo de la experiencia}

La entrada a la cueva es por un túnel (Figura 1), de manera que los niños/as desde el habitual pasillo de su colegio se adentran por el túnel hacia el interior de una cueva. Todo está oscuro y un fuerte viento se siente justo al entrar (ventilador dirigido hacia la boca del túnel). Se escuchan sonidos de animales, viento, lluvia, truenos y el mar, y pueden sentirse diferentes olores: las plantas aromáticas y un olor constante a incienso.

Dentro de la cueva, hay diferentes espacios: una zona en el centro donde los niños/as entran a probar, oler y tocar distintos alimentos (mandarina, arándanos, pomelo, moras, manzana, zanahoria, sal, azúcar), otra para manipular minerales con propiedades diferentes (forma, brillo, densidad, sabor), otra en la que pueden encontrar huesos de animales en el interior de unos arbustos (simulados con papel triturado), otra donde jugar con hojas secas, otra en la que hay un charco helado (simulado con hielos), una zona caliente donde está el fuego (simulado con almohadas de semillas calentadas al microondas y con una luz roja) y otra donde hay plantas como el romero aprovechando algún rayo de Sol que entra en la cueva (simulado por una linterna). Además, se prepararon botes con distintos olores (lavanda, menta), cestas con castañas, bellotas, conchas, plumas, piñas, utensilios de madera, palos, murciélagos de cartulina colgando del techo, y cubos con tierra y arena donde los niños/as pueden apreciar diferentes texturas con su mano. Por otra parte, de manera sincronizada, al sonar el agua se pulveriza con agua la sala y cuando se oye el relámpago se enciende instantáneamente una linterna. Introducir de manera lúdica materiales diversos permite al alumnado acceder a diferentes posibilidades de acción, descubrir lo que les gusta y lo que no les gusta, favorece el interés y la motivación y ayuda a desarrollar la espontaneidad y la creatividad (Sugrañes et al., 2012). 

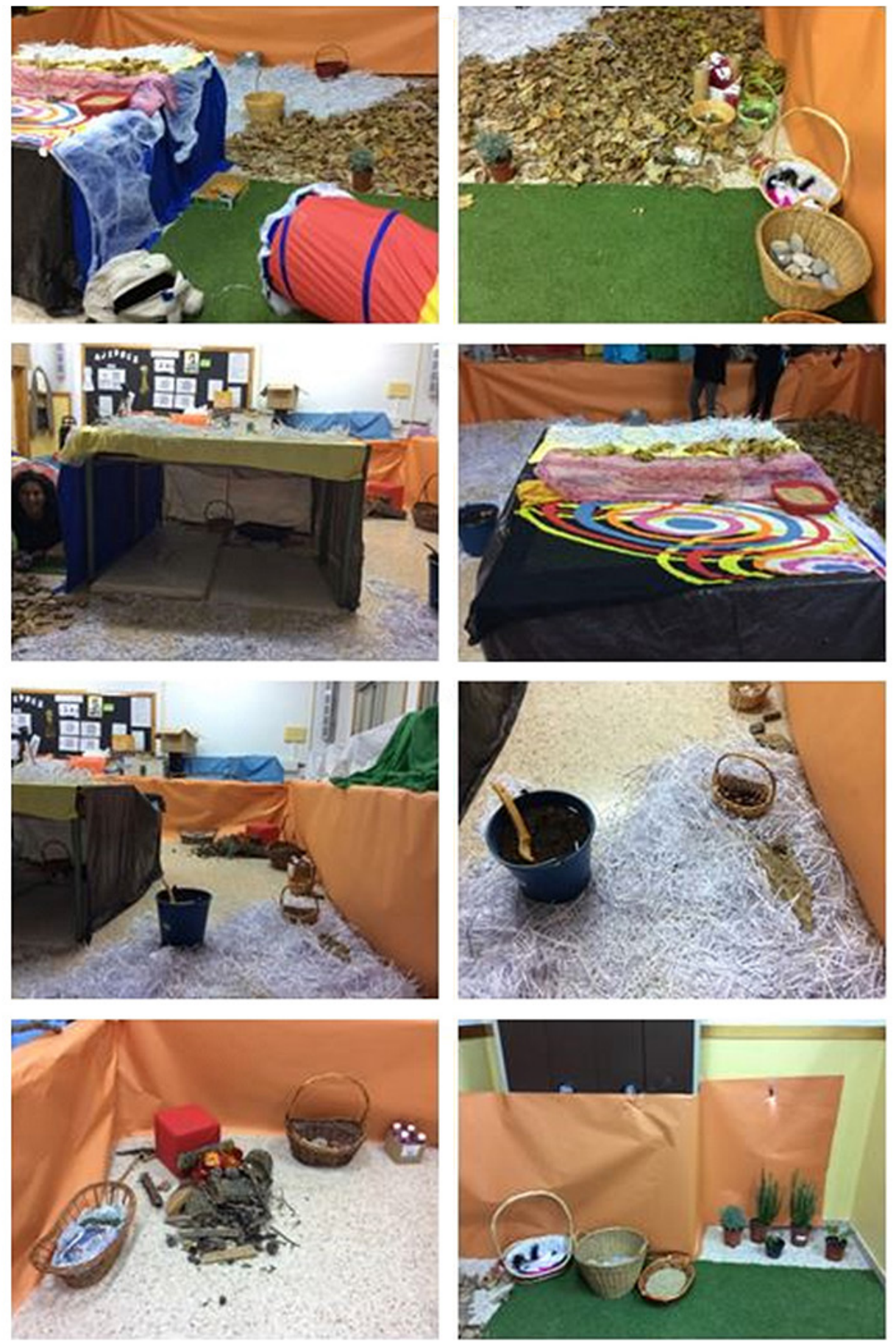

Figura 1. Espacios de la actividad multisensorial.

La sesión multisensorial consta de las siguientes fases: 1) 10 minutos a oscuras; 2) 5 minutos a oscuras donde cada niño/a explora con una linterna; 3) 3 minutos con luz y 4) 2 minutos de nuevo a oscuras. Durante toda la experiencia, las maestras acompañan a los niños/as en el aula animándoles a explorar y planteando preguntas sobre lo que hay en la cueva aunque son ellos los que exploran libremente el espacio con los diferentes recursos.

Todos los materiales utilizados están relacionados con la temática de la experiencia multisensorial, es decir, son olores, alimentos, sonidos, objetos y materiales que podríamos encontrar dentro de una cueva. Los elementos de la naturaleza son materiales que no 
pueden faltar en las aulas de El ya que son materiales transmisores de sensorialidad y, por tanto, fuentes de estímulo para fomentar la experimentación, la manipulación y la comunicación (Franco y Llinares, 2019). Estos materiales permiten la observación, comparación y clasificación sensorial de sus propiedades por parte de los niños/as, procedimientos científicos esenciales a trabajar en El (Fernández y Bravo, 2015).

\section{Resultados de la experiencia}

Al analizar la actividad multisensorial y su reflexión podemos deducir que todos los niños/ as utilizan y manifiestan haber utilizado los sentidos para explorar los materiales que había en el interior de la cueva: oído ("me han gustado los sonidos del perro y del pájaro", "he oído muchos sonidos"), gusto ("probar las moras", "he probado una cosa que era como una cereza y estaba muy rica"), tacto ("tocar las bellotas", "tocar las piedras", "tocar el frío", "encontrar un hueso y dárselo a Ángela para que lo toque"), vista ("encontrar con las linternas los huesos", "cuando estábamos con las linternas veía todo") y olfato ("cuando olía las cosas").

Tanto al observar la experimentación libre de los niño/as dentro de la cueva como en sus explicaciones y descripciones en la sesión posterior, se manifiesta que todas las percepciones van ligadas a una o varias emociones. Las emociones que surgieron en el alumnado fueron entusiasmo, curiosidad, nerviosismo ante lo desconocido, sorpresa positiva, miedo ("cuando he pasado por el túnel me ha hecho gracia, pero tenía un poco de miedo pero luego me he metido y he superado el miedo"), alegría ("he sentido alegría porque he cogido una linterna y he encontrado un hueso"), etc.

Las dos maestras por su parte reconocen que es necesario realizar mayor número de actividades donde se ejerciten los sentidos explícitamente (“...vivimos en una sociedad visual y rápida que nos hace desarrollar menos el resto de los sentidos. El aprendizaje se verá beneficiado cuanto más desarrollemos todos los sentidos") y, trabajar a su vez, vocabulario sensorial que ayude a los niños/as a describir aquello que están explorando. Apuntan que se han dado cuenta de la poca experiencia que tenían al trabajar los sentidos con su alumnado. Estas afirmaciones son de gran valor a nivel educativo, ya que son el punto de partida para que propongan más actividades de este tipo en el futuro. Reconocen el imprescindible papel del profesor para guiar y acompañar al alumnado en este tipo de actividades ("el adulto dirige, planifica y organiza, pero siempre acompañando y dejando hacer a los niños, lo que implica no anticiparse al niño") y las dificultades que han surgido al llevar a cabo la actividad: temporales ("necesitan más tiempo para explorar") y didácticas ("nos falta vocabulario sensorial para poder hacer preguntas a los alumnos", "es difícil no dirigir a los niños", etc).

Ante la demanda de las maestras, en la tabla 1 se proponen algunos conceptos que se podrían trabajar de manera previa, durante y posterior haciendo alusión a cada uno de los sentidos en experiencias de Ciencias en las aulas de El. Tras la reflexión de las maestras consideramos oportuno incluir una tabla de vocabulario sensorial como herramienta concreta para trabajar la educación sensorial. Así, los niños/as serán capaces de describir de manera más completa los materiales y las sensaciones en otras situaciones futuras.

Esta tabla sirvió como material didáctico de apoyo a las maestras para aplicarlo al proyecto en situaciones cotidianas para trabajar la educación sensorial. Por ejemplo, la semana siguiente a la actividad, las maestras aprovecharon un día de lluvia para salir al patio con su alumnado y utilizar los 5 sentidos: sentir las gotas de lluvia en sus caras, oler el jardín, observar el cielo, disfrutar del sonido de la lluvia, jugar y tocar el agua y, a su vez, a compartir las experiencias verbalizando lo que estaban sintiendo (Figura 2). 
Tabla 1. Características de las tablas y descripción de las mismas.

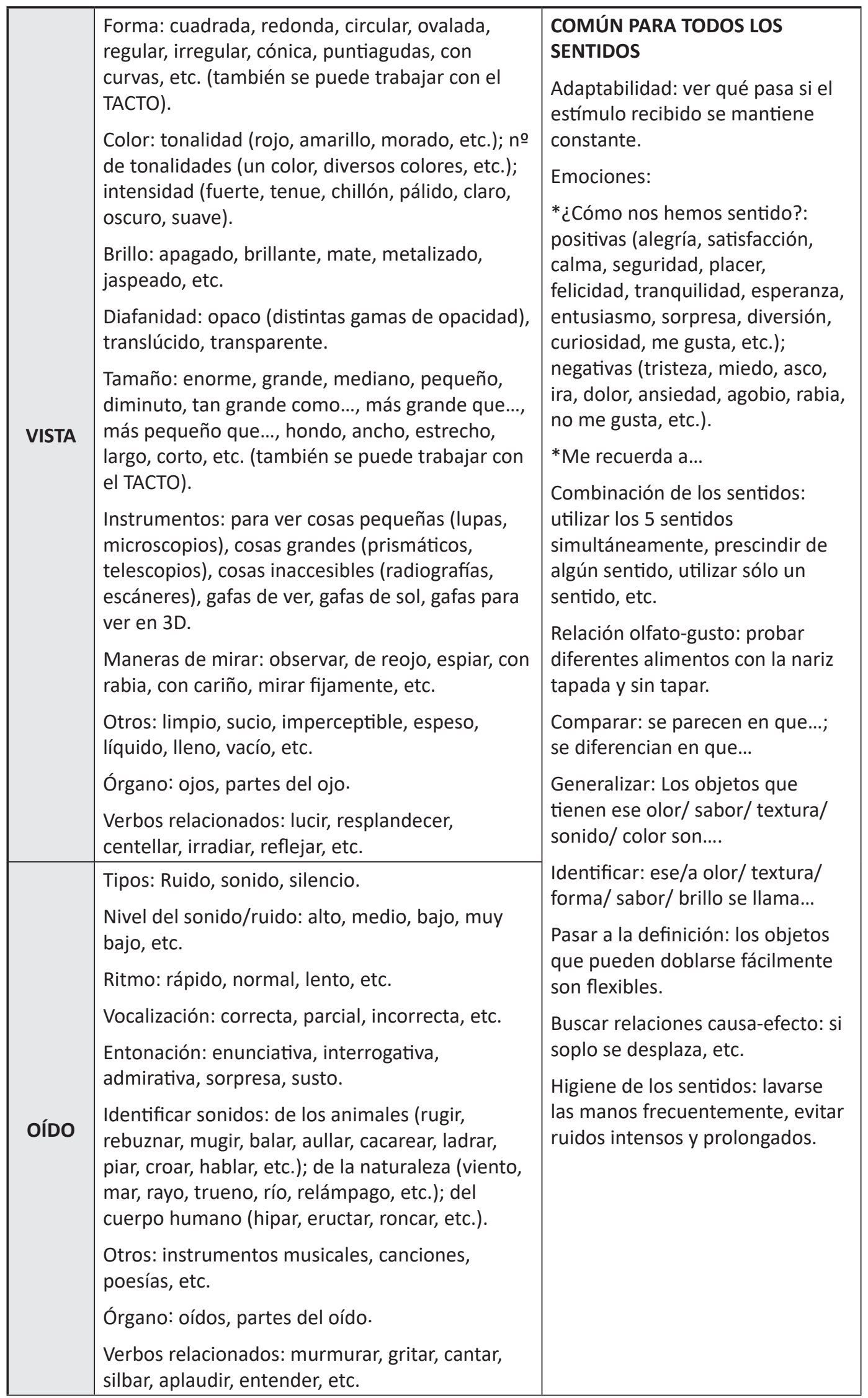


Tabla 1. Características de las tablas y descripción de las mismas. Continuación

\begin{tabular}{|c|c|}
\hline TACTO & $\begin{array}{l}\text { Texturas: arrugado, terso, elástico, frágil, flexible, } \\
\text { granuloso, harinoso, pastoso, untuoso, áspero, } \\
\text { fluido, rugoso, liso, fino, aterciopelado, con } \\
\text { protuberancias, con relieve, con incisiones, con } \\
\text { agujeros, pulido, poroso, apelmazado, sedoso. } \\
\text { Consistencia: duro, blando, compacto, pesa, no } \\
\text { pesa. } \\
\text { Humedad: mojado, empapado, húmedo, seco. } \\
\text { Temperatura: congelado, frio, frescos, templado, } \\
\text { tibio, caliente, etc. } \\
\text { Órgano: piel, partes de la piel. } \\
\text { Verbos relacionados: tocar, sentir, pinchar, } \\
\text { raspar, hacer cosquillas, abrazar, besar, recibir } \\
\text { caricias, dar la mano, pellizcar, etc. }\end{array}$ \\
\hline GUSTO & $\begin{array}{l}\text { Tipos: dulce, salado, ácido, amargo, picante, } \\
\text { insípido. } \\
\text { Intensidad: ligero, equilibrado, intenso, etc. } \\
\text { Regusto: persistente, pronunciado, tiene } \\
\text { continuidad, instantáneo. } \\
\text { Otros: jugoso, blando, duro, crujiente, pegajoso, } \\
\text { granuloso, pastoso, podrido, con especias, } \\
\text { extraño, sorprendente, exótico, exquisito, } \\
\text { original, sabroso, etc. } \\
\text { Órgano: lengua, partes de la lengua. } \\
\text { Verbos relacionados: comer, beber, absorber, } \\
\text { soplar, saborear, cenar, almorzar, etc. }\end{array}$ \\
\hline OLFATO & $\begin{array}{l}\text { Tipos: Odoríferos (emiten olor) o inodoros (no } \\
\text { emiten olor) } \\
\text { Según su procedencia: animal (ámbar gris), } \\
\text { vegetal (romero), mineral (azufre), ambientales } \\
\text { (panadería, pescadería), artificial. } \\
\text { Tipos de olor: agradable, aromático, mentolado, } \\
\text { resinoso, agrio, fragante, floral, pútrido, rancio, } \\
\text { quemado, etéreo, fragante, repugnante, } \\
\text { nauseabundo, a cerrado, a humedad, a } \\
\text { naturaleza, nuevo, familiar, puro, combinado, a } \\
\text { chamusquina, etc. } \\
\text { Órgano: nariz, partes de la nariz. } \\
\text { Verbos relacionados: oler, olisquear, perfumar, } \\
\text { atufar, aromatizar, etc. }\end{array}$ \\
\hline
\end{tabular}




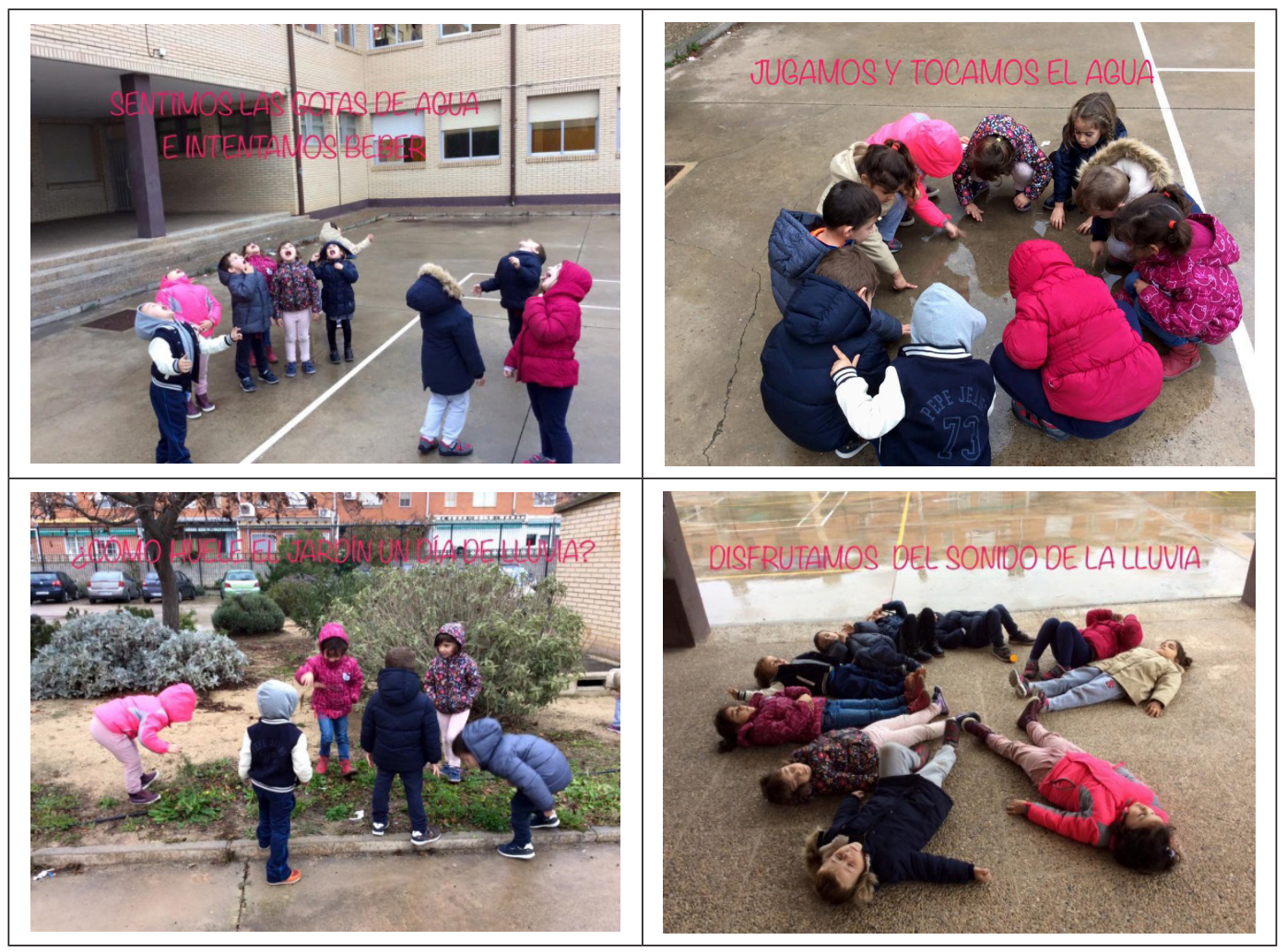

Figura 2. Educación sensorial en un día de lluvia.

\section{Conclusiones e implicaciones educativas}

Atendiendo a los dos primeros objetivos, podemos señalar que al diseñar e implementar esta experiencia multisensorial se han trabajado aspectos científicos a través de los sentidos. Por ejemplo, a partir del uso del lenguaje en la descripción de materiales y sensaciones. Al igual que Gutiérrez (1998) consideramos que trabajar el lenguaje conjuntamente con el conocimiento científico desde los inicios es crucial, ya que no hay ciencia sin palabras para referirse a ella, pero tampoco sin palabras para comunicarla. Dicha experiencia sirvió como base para comenzar un proyecto concreto (la luz) que se desarrolló a lo largo del curso, con contenidos y procedimientos científicos que se empezaron a utilizar en esta sesión.

El tercer objetivo, relacionado con la reflexión de las maestras se considera satisfecho, ya que de manera paralela se han aportado estrategias a los maestros/as para trabajar la educación sensorial en la realidad escolar: aprovechando fenómenos cotidianos del aula, o fenómenos meteorológicos, del cambio de las estaciones, etc. y provocando situaciones para centrar la atención en un sentido concreto, y de varios al mismo tiempo; e introduciendo diversos materiales para progresar en sus habilidades de exploración, aumentando las percepciones y tomando conciencia de ellas; o planteando diálogos reflexivos que sirvan para compartir e interiorizar las experiencias. De esta manera, introducimos a los niños en el mundo de la ciencia, con un acercamiento al mundo natural a partir de una experiencia desarrollada en el interior de una "cueva", desarrollando su motivación por explorar sensorialmente a la vez que disfrutan de los descubrimientos.

Es necesario establecer colaboraciones continuadas y fluidas entre Universidad-escuela que permitan contribuir a la mejora del aprendizaje profesional de los docentes y, consecuentemente, al desarrollo preescolar (Elm y Nordqvist, 2019). Además, desde el campo 
de la Didáctica de las Ciencias Experimentales esta colaboración es fundamental para detectar necesidades y expectativas de las escuelas y para que los hallazgos de la investigación lleguen a las aulas.

\section{Agradecimientos:}

A Alicia, Amparo y a los niños.

Financiado por el Ministerio de Economía y Competitividad (proyecto EDU2016-76743-P), así como por el Gobierno de Aragón (Grupo de investigación BEAGLE, perteneciente al Instituto Universitario de Investigación de Ciencias Ambientales de Aragón, IUCA) y cofinanciado con Feder 2014-2020.

\section{Bibliografía}

Borgerding, L.A. y Raven, S. (2018). Children's ideas about fossils and foundational concepts related to fossils. Science Education, 102, 414-439. DOI: https://doi.org/10.1002/ sce. 21331

Cantó Doménech, J., de Pro, A. y Solbes, J. (2016). ¿Qué ciencias se enseñan y cómo se hace en las aulas de educación infantil? La visión de los maestros en formación inicial. Enseñanza de las Ciencias, 34(3), 25-50. DOI: https://doi.org/10.5565/rev/ ensciencias.1870

De Puig, I. (2004). Persensar. Percibir, sentir, pensar. Barcelona: Octaedro.

Díez Navarro, M.C. (2013). 10 ideas clave. La educación infantil. Barcelona: Graó.

Driver, R.,Guesne, E. y Tiberguien, A. (1989). Ideas científicas en la infancia y en la adolescencia. Madrid: MEC y Morata.

Elm, A. y Nordqvist, I. (2019).The research circle - a tool for preschool teachers' profesional learning and preschool develpment. European Journal of Teacher Education, 42(5), 621-633. DOI: https://doi.org/10.1080/02619768.2019.1652899

Fernández Manzanal, R. y Bravo Tudela, M. (2015). Las ciencias de la naturaleza en Educación Infantil. El ensayo, la sorpresa y los experimentos se asoman a las aulas. Madrid: Ed. Pirámide.

Franco, J.P. y Llinares, C. (2019). Materiales en la escuela infantil 0-3: objetos que tejen la vida cotidiana. Aula de Infantil, 101, 13-16.

Gardner, H. (2005). Inteligencias múltiples. La teoría en la práctica. Madrid: Paidós.

García-Carmona, A., Criado, A.M. y Cañal, P. (2014). Alfabetización científica en la etapa 3-6 años: un análisis de la regulación estatal de enseñanzas mínimas. Enseñanza de las Ciencias, 32(2), 131-149. DOI: https://doi.org/10.5565/rev/ensciencias.817

Gutiérrez, B. (1998). La ciencia empieza en la palabra. Barcelona: Península.

Hernández-Sampieri, R., Fernández, C. y Baptista, P. (2006). Metodología de la investigación. México D.F.: McGraw-Hill/Interamericana.

Mazas, B., Gil-Quílez, M.J., Martínez-Peña, B., Hervas, A. y Muñoz, A. (2018). Los niños y las niñas de infantil piensan, actúan y hablan sobre el comportamiento del aire y del agua. Enseñanza de las Ciencias, 36(1), 163-180. DOI: https://doi.org/10.5565/rev/ ensciencias. 2320 
Mellado, V., Borrachero, A. B., Brígido, M. y Melo, L.V. (2014). Las emociones en la enseñanza de las ciencias. Enseñanza de las Ciencias, 32(3), 11-36. DOI: https://doi. org/10.5565/rev/ensciencias.1478

Montiera, S.F. y Jiménez-Aleixandre, M.P. (2016). The practice of using evidence in kindergarten: the role of purposeful observation. Journal of Research in Science Teaching, 55(8), 1232-1258. DOI: https://doi.org/10.1002/tea.21259

Real Decreto 1085/2008, de 28 de marzo, por la que se aprueba el currículo de la Educación Infantil y se autoriza su aplicación en los centros docentes de la Comunidad Autónoma de Aragón. Boletín Oficial de Aragón, de 14 de abril de 2008.

Sugrañes, E., Alós, M., Andrés, N., Casal, S., Castrillo, C., Medina, N. y Yuste, M. (2012). Observar para interpretar. Actividades de vida cotidiana para la educación infantil (2-6). Barcelona: Grao.

Vega, S. (2012). Ciencia 3-6. Laboratorios de ciencias en la escuela infantil. Barcelona: Grao.

Vila, B. y Cardo, C. (2012). Material sensorial (0-3 años). Manipulación y experimentación. Barcelona:Grao.

Westerhoff, N. (2010). La neurodidáctica a examen. Mente y cerebro, 44, 34-40. 
